changes during HF remodelling, and is an inaccurate representation of cardiac function. We further showed that this is because EF is calculated using the endocardial boundary rather than the mid-wall layer, because a corrected EF parameter (CEF) that uses the mid-wall layer for quantification resolves this shortcoming. This CEF became independent of cardiac geometry, and could successfully distinguish HFpEF and healthy heart in our animal models, where EF could not. The CEF can be calculated easily with measurements of EF, wall thickness, and LV inner diameter. We further showed that the myocardial strains at the endocardial and epicardial boundary deviated significantly from each other and from the strains at the mid-wall layer, and this magnitude of deviation depended on the cardiac geometry, suggesting that any quantification of cardiac function using the epi- or endo- boundaries can be skewed by geometric changes to the heart, and will be ineffective. Finally, we tested specific types of HF morphologies, namely, eccentric hypertrophy, concentric hypertrophy, and concentric remodelling with our numerical model. We found that the concentric remodelling morphology is the most inefficient in converting myocardial strains to stroke volume, while eccentric hypertrophy is the most efficient. This may explain why HFpEF hearts, which are likely have wall thickening similar to concentric remodelling, have exercise intolerant, and our results suggest that cardiac dilation during HFrEF is providing advantages to help with flow function.

Conclusion We demonstrated that geometric changes during $\mathrm{HF}$ remodeling can significantly impact cardiac function, and can skews functional parameters like EF. We further showed that the reason for EF's shortcoming is due to a flawed reliance on quantification using the endocardial boundary of the heart rather than the mid-wall layer. We proposed a new CEF parameter to replace EF that can resolve the shortcoming, and that can distinguish HFpEF from healthy hearts.

Conflict of Interest None to declare

\section{WHAT ACTUALLY HAPPENS IN A SPECIALIST HEART FAILURE CLINIC?}

${ }^{1}$ Arvind Singhal, 'Jiliu Pan, 'Barbara Cassimon, ${ }^{2}$ Martin Cowie. 'Royal Brompton Hospital, London, UKi ${ }^{2}$ Imperial College, London

\subsection{6/heartjn-2021-BCS.133}

Introduction Provision of specialist outpatient services has struggled to keep up with the growing demands of the heart failure (HF) epidemic in the UK. The Covid-19 pandemic necessitated a dramatic change in how HF clinics are delivered, with a reduction in face-to-face contact. The need for in-person appointments remains unknown. We evaluated prepandemic face-to-face clinic appointments in detail, to see what appointments achieved, in order to plan future services. Methods We conducted a retrospective cohort study of patients who had completed 3 years of follow up (1st January 2017 to 31st December 2019) in a specialist HF clinic. Inclusion criteria were a clinician diagnosis of HF or left ventricular systolic dysfunction (from clinic letters and echocardiography), or at least moderate valvular disease with symptoms. Patient electronic health records were reviewed, and for each clinic attendance, the following were noted: - Presence of new or worsening symptoms or signs of HF, or symptoms relating to HF treatment, as assessed by the clinician $\bullet$ Change in cardiovascular medications $\bullet$ Investigations requested $\bullet$ Documented patient education, advice, or discussion of care plan

Results 100 patients were included in the cohort. The median age was 68 , and patients were predominantly male $(80 \%)$ with HFrEF (78\%). There was a total of 666 appointments over the 3 years of follow up. Patients had a median of 2 appointments per year (Figure 1). Figure 2 shows the frequency of investigations, symptoms and clinician actions in appointments. $21 \%$ of appointments documented new or worsening HF or treatment-related symptoms, and cardiovascular therapy was changed in $36 \%$ of appointments, ranging from $55 \%$ of nurse-led appointments to $31 \%$ where the patient saw a junior doctor. Therapy change was more common in appointments when there was a change in symptoms $(53 \%$ vs $31 \%$, p < 0.001$)$. $41 \%$ of patients had no documented worsening in HF symptoms throughout the entire 3 year follow up period. Patient education or clinical advice was documented in $32 \%$ of appointments. Follow-up duration was shorter following a change in symptoms (127 vs 163 days, $\mathrm{p}=0.03$ ) and therapy change (128 vs 170 days, $\mathrm{p}=0.03) .45 \%$ of appointments had no documented worsening HF symptoms, change in cardiovascular therapy, specialist referral or specialist test (excluding echocardiography and phlebotomy); we consider these to be 'routine' follow-up appointments.

Conclusion Most HF patients were seen in a specialist HF clinic at least 6-monthly, but worsening symptoms and therapy change at the time of consultation were uncommon. Investigations other than phlebotomy and echocardiography, which may be performed outside of specialist centres, were rarely

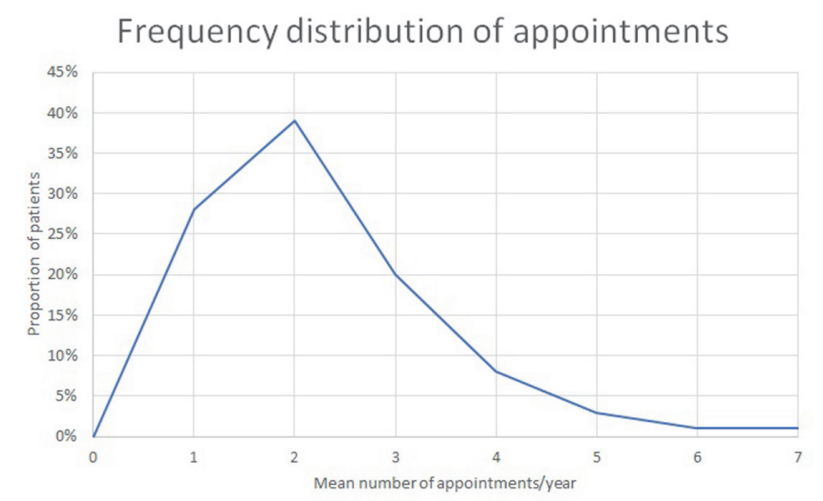

Abstract 136 Figure 1

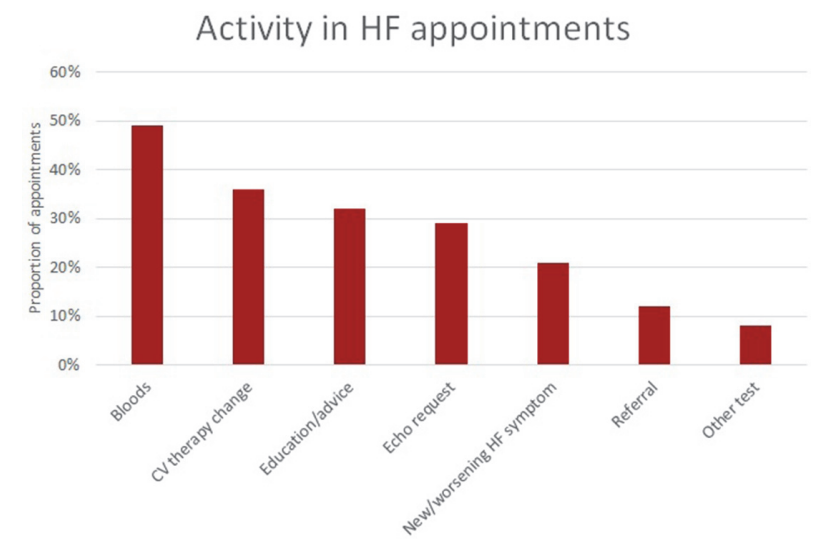

Abstract 136 Figure 2 
performed. Many appointments may therefore be suitable for community services or by telemedicine, thus freeing up capacity and responsiveness of in-person specialist clinics for patients with more complex needs, worsening symptoms or new diagnoses.

Conflict of Interest Dr Singhal's salary is funded by a fellowship from Abbott

\section{IS A DEDICATED HFPEF CLINIC EFFECTIVE IN MANAGING PATIENTS WITH HEART FAILURE WITH PRESERVED FRACTION (HFPEF)?}

Patrick Tran, Thomas Long, Jessica Smith, Prithwish Banerjee. University Hospitals Coventry and Warwickshire, Coventry, UK

\subsection{6/heartjnl-2021-BCS.134}

Background HFpEF is often diagnosed in elderly patients coupled with comorbidities and degrees of frailty. Given the lack of evidence-based therapies, the common maxim is to 'optimize comorbidities with a holistic approach,' Joint cardiology-geriatric HFpEF clinics have been recommended but are unlikely to be sustainable with current resource constraints. To improve outcome a dedicated HFpEF clinic led by HF specialists was therefore piloted to include specific interventions: diuretics, fluid restriction $(<1.5 \mathrm{~L} /$ day $)$, heart rate control $(<70 /$ min for sinus and $<80 / \mathrm{min}$ for atrial fibrillation (AF)), blood pressure (BP) control $(<130 / 80 \mathrm{mmHg})$, cardioversion for $\mathrm{AF}$, exercise recommendation and control of non-cardiovascular comorbidities (e.g. diabetes, COPD, obesity).

Methods A single-centre retrospective analysis of $202 \mathrm{HFpEF}$ out-patients was performed over a 12-month period. Diagnosis of HFpEF was based on a local diagnostic algorithm similar to the HFA-PEFF score recommended by ESC. Electronic and paper records were reviewed, capturing comorbidities, clinic interventions and follow-up. We also performed a pilot analyses of hospitalization and mortality rates between patients managed in the general HF clinic and in the dedicated HFpEF clinic.

Results The study population reflected a typical HFpEF profile (mean age $79 \pm 9.6$ years, 55\% female, NYHA Class III/IV (48\%), hypertension (78\%), AF, 53\%, chronic kidney disease (41\%), ischaemic heart disease (30\%) and obesity (13\%) (table 1). Clinic time was limited to 15-20 minutes. The majority of clinic interventions were cardiology-focused, namely congestion management (64\%), drug reviews (86\%) of anti-hypertensives, and rate/rhythm control of AF (48\%). With the current outpatient structure, there was little remaining time to consider non-cardiac comorbidities. Although not powered in the pilot analysis, preliminary data showed no difference in hospitalization and mortality rates between general $\mathrm{HF}$ and dedicated HFpEF clinics. In both clinics, $25 \%$ of patients had at least two admissions at 12 months mainly due to non-cardiac causes (table 2).

Conclusion Structure of specialized HFpEF clinics needs to be reshaped to meet the complex demands of HFpEF and improve patient outcomes. Current clinic activities concentrated on managing fluid balance, $\mathrm{BP}$ and cardiac drug reviews with little emphasis on comorbidities e.g. optimizing diabetes, anaemia or addressing polypharmacy which are common causes for re-hospitalization. We believe that our outpatient clinic reflects most HF clinics in the UK. Accordingly, an efficient HFpEF clinic pathway needs to be formulated. Quality improvement ideas include (1) screening patients before clinic for any significant comorbidities to enable early specialist advice or referral (2) entrusting HF nurse specialists to

Abstract 137 Table 1 Baseline characteristics of patients

\begin{tabular}{|c|c|c|c|}
\hline Demographics & $\begin{array}{l}\text { HFpEF clinic } \\
(\mathrm{N}=101)\end{array}$ & $\begin{array}{l}\text { General clinic } \\
\qquad(N=101)\end{array}$ & P Value \\
\hline Age- year (mean $\pm \mathrm{SD})$ & $78 \pm 9.8$ & $79 \pm 9.4$ & 0.38 \\
\hline Female sex- no (\%) & $57(56.4)$ & $55(54.5)$ & 0.89 \\
\hline Average number of clinic visits (IQR) & $1(1-2)$ & $1(1-2)$ & 0.07 \\
\hline \multicolumn{4}{|l|}{ Number of clinic visits } \\
\hline 1 & 68 & 56 & \\
\hline 2 & 24 & 29 & \\
\hline 3 & 6 & 14 & \\
\hline 4 & 3 & 1 & \\
\hline 5 & 0 & 1 & \\
\hline \multicolumn{4}{|l|}{ Co-morbidities- no (\%) } \\
\hline Hypertension & $78(77.2)$ & $79(78.2)$ & 0.87 \\
\hline Coronary artery disease & $28(27.7)$ & $33(32.7)$ & 0.44 \\
\hline Atrial fibrillation & $51(50.5)$ & $56(55.4)$ & 0.48 \\
\hline Type II Diabetes (T2DM) & $31(30.7)$ & $36(35.6)$ & 0.46 \\
\hline Valvular heart disease & $25(24.8)$ & $20(19.8)$ & 0.40 \\
\hline Chronic kidney disease (estimated GFR<60) & $38(37.6)$ & $44(43.6)$ & 0.39 \\
\hline Chronic obstructive pulmonary disease & $11(10.9)$ & $11(10.9)$ & 1.00 \\
\hline Obesity (BMI $\geq 30$ ) & $17(16.8)$ & $10(9.9)$ & 0.15 \\
\hline \multicolumn{4}{|l|}{ NYHA functional class- no (\%) } \\
\hline I & 5 & 6 & 1.00 \\
\hline II & 53 & 42 & 0.17 \\
\hline III & 39 & 53 & 0.07 \\
\hline IV & 4 & 0 & 0.12 \\
\hline
\end{tabular}

